\title{
RADIOGRAPHIC APPEARANCES IN LUMBAR DISC PROLAPSE
}

\author{
J. G. B. MACLEAN, J. K. TUCKER, J. B. LATHAM
}

From Norfolk and Norwich Hospital, Norwich

\begin{abstract}
The pre-operative lumbar spine radiographs of 200 consecutive patients who had undergone discectomy for prolapsed intervertebral disc were reviewed. Prolapse was recognised as bulging or sequestration of the disc with consequent root compromise.

Measurement of the lumbar level of the interiliac line was shown to correlate with the level of disc prolapse and the incidence of transitional vertebrae at the lumbosacral junction was significantly higher than normal. A pathological value for the lumbosacral angle could not be identified.
\end{abstract}

Many radiological surveys comparing patients with and without backache have failed to demonstrate any significant difference between the two groups (Splithoff 1953; Witt 1984). Likewise massive pre-employment screening programmes have failed to identify the 'at risk' spine by plain radiography (Rowe 1982). Where disc prolapse is the only suspected pathology, the likely benefit of a plain radiograph has been questioned, other than as a preliminary to myelography or to determine dermatome levels accurately (McCulloch and Waddell 1980; Wigh 1980).

In this present study we concentrated on three parameters detectable on a plain radiograph; the lumbar level of the interiliac line, the incidence of transitional vertebrae at the lumbosacral junction, and the lumbosacral angle. Little work has been done on patients with proven disc prolapse, and it was hoped that by studying such a population it might be possible to relate certain plain film characteristics with the established disc pathology.

MacGibbon and Farfan (1979) suggested that if the lumbar level of the interiliac line was high then L5 would be deep-seated in the pelvis, and the L5/S1 disc space relatively protected. In such cases they predicted that, should prolapse occur, it would be more likely at the L4/ 5 rather than the L5/S1 disc. Alternatively, when the

J. G. B. MacLean, FRCS, Orthopaedic Registrar

J. K. Tucker, FRCS, Consultant Orthopaedic Surgeon

J. B. Latham, FRCR, Consultant Radiologist

Norfolk and Norwich Hospital, Brunswick Road, Norwich NR1 3SR, Norfolk, England.

Correspondence should be sent to Mr J. G. B. MacLean at 7 Woodlands Avenue, Wanstead, London E11 3RA, England.

(C) 1990 British Editorial Society of Bone and Joint Surgery 0301-620X/90/5153\$2.00

J Bone Joint Surg [Br] 1990; 72-B :917-20. interiliac line was low they regarded L5 as high-riding and less protected, so that prolapse would be more likely at the L5/S1 level. Their work, based on biomechanical principles, was purely theoretical, in that their predictions resulted from a radiological survey of patients who had never been admitted to hospital for backache.

The second parameter we studied was the bony configuration of the lumbosacral junction, noting the presence of transitional vertebrae. Previous authors (Splithoff 1953; Fullenlove and Williams 1957; Tini, Wieser and Zinn 1977) have questioned the relevance of such anomalous vertebrae, although it has been suggested that they may alter the biomechanics of the lumbosacral region (Mitchell 1936). In the literature, different rates of detection have been reported with conflicting conclusions. A comparison of these studies is not really valid as the criteria for recognition have not been uniform and, in some cases, have not been discussed.

In a study of the incidence, prevalence and significance of transitional vertebrae, Tini et al (1977) described a clear and reproducible radiological classification. However, computer analysis correlating the radiological findings with the age, sex and symptomatology of 798 patients (Zinn 1972) failed to show any relation between these variables and the incidence of transitional vertebrae. The incidence he noted $(7.9 \%)$ showed minor geographical variation when compared with other surveys he reviewed, but was not significantly different from those reported by previous authors (Splithoff 1953; Fullenlove and Williams 1957).

The third parameter we studied was the lumbosacral angle. It has been suggested that an increased lumbosacral angle may predispose to backache. Von Lackum (1924) showed that at an angle of $35^{\circ}$ the pressure tending to slide the penultimate on the ultimate vertebra was $0.57 \%$ of the total body-weight; this increased to $0.97 \%$ at an 
angle of $75^{\circ}$. Thus it would seem reasonable to suspect an association between the angle and disc pathology. It has, however, proved difficult to differentiate what is abnormal in a parameter with such a wide range of values, and there are many who doubt its clinical significance (Bene 1981). We have attempted to identify a pathological value for this parameter.

\section{PATIENTS AND METHODS}

All patients who had undergone surgery in the Norfolk and Norwich Hospital for presumed prolapsed intervertebral disc between 1981 and 1986 were reviewed. The first 200 with complete case notes, adequate radiographs and unequivocal prolapse treated by discectomy alone were selected. Disc prolapse was recognised as bulging or sequestration of the disc with consequent root compromise.
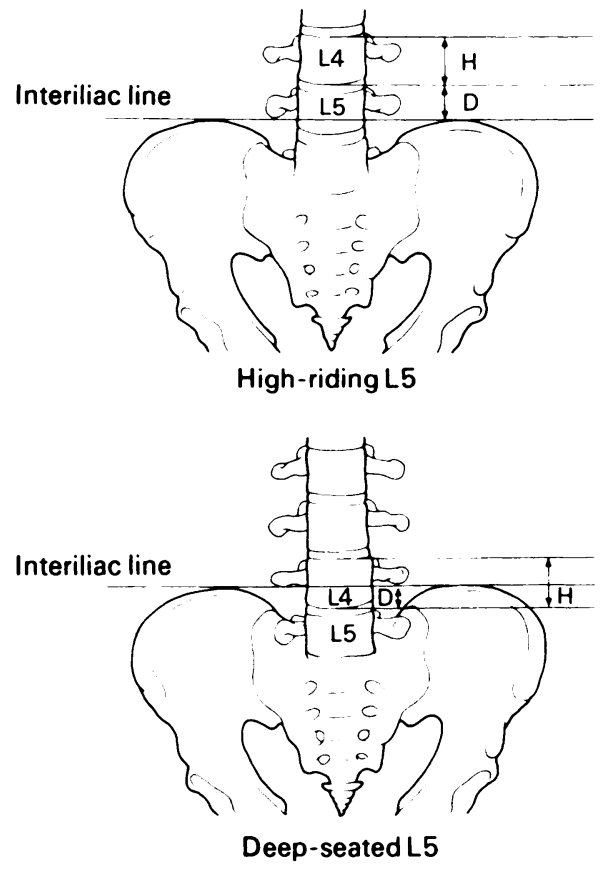

Fig. 1

The lumbar level of the interiliac line.

The interiliac line. The pre-operative plain films were reviewed and the point at which a line drawn between the iliac crests bisected the lumbar spine was measured from the lower border of L4 (Fig. 1). We expressed this value (D) as a fraction of the vertebral height of $\mathrm{L} 4(\mathrm{H})$, thus allowing for variation in patient size and radiographic magnification. When $\mathrm{D}$ was less than $10 \%$ of the vertebral height of $\mathrm{L4}$, whether above or below, we regarded $L 4$ as being on the interiliac line. When the lower border of L4 was above the interiliac line
(D $>10 \%$ ) L5 was regarded as high-riding, and when below (D > 10\%) L5 was regarded as deep-seated.

It is accepted that the interiliac line cannot be measured precisely and that some degree of parallax may occur; however, we felt that the $10 \%$ margin we allowed compensated for this. A pilot study was conducted to determine to what extent assessment of the lumbar level of the interiliac line was influenced by radiographic factors.

A series of radiographs were taken using an articulated lumbar spine and pelvis; once in place the bones were not moved. One radiographic factor was varied for each group of films, and the relationship of the lumbar level of the interiliac line to the lower border of L4 was observed. The limits of each group were designed to cover even the most extreme of radiographic faults and demonstrated the surprisingly small influence which radiographic factors have on this assessment. Variation of the centre point of the film in the horizontal or vertical plane also had minimal effect, as did alteration of the tube-film distance. Angulation of the tube did appear to affect measurement of the distance $D$, an angulation of $20^{\circ}$ altering the measurement by $28 \%$ of the vertical height of L4.

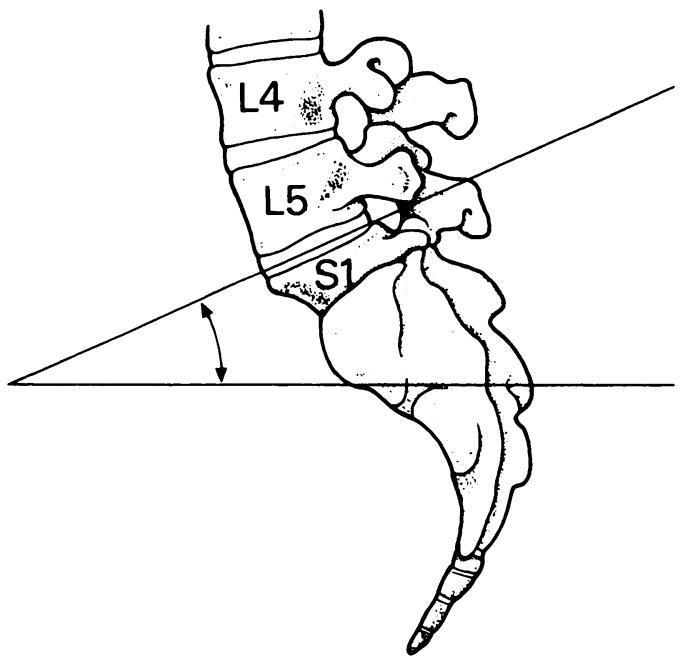

Fig. 2

The lumbosacral angle.

Transitional vertebrae. From the anteroposterior film the bony configuration of the lumbosacral junction was scrutinised and, applying the criteria described by Tini et al (1977), the incidence of transitional vertebrae was recorded. His classification, based on radiomorphological changes of the transverse processes, recognised four groups.

1. Dysplasia of the transverse process of the last presacral vertebra, subdivided into la asymmetrical, and $1 \mathrm{~b}$ symmetrical. 
2. Symmetrical transitional vertebrae: (a) with fusion between the transverse process and sacral wings; (b) with elongation of the transverse process towards the sacral wings with formation of new articulations without complete bridging.

3. Asymmetrical transitional vertebrae: (a) a combination of fusion on one side and articulation on the other; (b) fusion on one side with a normal transverse process on the other; (c) articulation on one side with a normal transverse process on the other.

$\begin{aligned} & \text { Table I. Number of cases of proved disc } \\
& \text { prolapse at lowest two lumbar levels }\end{aligned}$
\begin{tabular}{lll}
\hline $\begin{array}{l}\text { Level of } 15 \text { in relation to } \\
\text { interiliac line }\end{array}$ & LA/5 & L5/S1 \\
\hline Deep-seated & 60 & 38 \\
High-riding & 21 & 39 \\
Neither & 16 & 22 \\
\hline
\end{tabular}

4. A combination of a dysplastic transverse process on one side and articulation or fusion on the other.

The terms lumbarisation and sacralisation were deliberately avoided since the thoracic vertebrae were not always visualised and consequently positive identification of the fifth lumbar vertebra was not possible. Spina bifida occulta and other posterior arch dysplasias were not included.

The lumbosacral angle. The angle we measured was that between the upper surface of the last fully mobile vertebra and the horizontal (Fig. 2). This was measured from the lateral film taken with the legs extended, so that the longitudinal axis of the patient would form a right angle with the bottom margin of the film, as described by Splithoff (1953).

\section{RESULTS}

In the population of 128 men and 72 women we studied there was no significant age difference between the sexes, the average being 39.4 years (range 17 to 70 ). As expected, prolapse occurred most frequently at the lowest two levels : 97 patients had prolapse at L4/5, 99 at L5/S1, and four at $\mathrm{L} 3 / 4$.

Table I shows the relationship between the level of disc prolapse and the lumbar level of the interiliac line. As can be seen, a deep-seated L5 was associated with a higher likelihood of prolapse at $\mathrm{L} 4 / 5$, whereas a highriding $\mathrm{L} 5$ was associated with prolapse at $\mathrm{L} 5 / \mathrm{S} 1$. Both of these associations were highly significant $(p<0.005)$.

Table II compares the incidence of lumbosacral transitional vertebrae in our series with that observed in a population who had never been admitted to hospital for backache. We found a significantly higher incidence of such bony anomalies than reported by Tini et al 1977 $(p<0.005)$.

The lumbosacral angle in our series measured on average $31.9^{\circ}$ (range $3^{\circ}$ to $57^{\circ}$ ). This was not significantly different from other reported values.

\section{DISCUSSION}

Our study has confirmed the hypothesis, based on biomechanical theory, that the lowest lumbar disc is protected from prolapse when it is deep-seated in the pelvis; when it is high-riding the lumbosacral disc is the likely site of prolapse. These findings suggest that the lowest 'unprotected' level is subject to greater mechanical stresses than higher levels. Thus from the lumbar level of the interiliac line one can anticipate the level of disc prolapse should this occur.

Table II. Incidence of lumbosacral transitional vertebrae (LTV), number and per cent

\begin{tabular}{lll}
\hline Population & $\begin{array}{l}\text { Our series } \\
(\mathbf{n}=\mathbf{2 0 0})\end{array}$ & $\begin{array}{l}\text { Normals* } \\
(\mathbf{n}=\mathbf{7 9 8})\end{array}$ \\
\hline Without LTV & 129 & 720 \\
With LTV (all groups) & 7135.5 & 789.7 \\
With LTV group I only & 4924.5 & 344.2 \\
With LTV group II only & 2211 & 445.5 \\
\hline
\end{tabular}

*Tini et al (1977) series

The significance of transitional vertebrae has been debated. It is unclear whether they result in increased, decreased or asymmetrical movement in their vertebral segment. Previous studies of these developmental anomalies have rarely specified their diagnostic criteria, but have stated that there is little or no association between transitional vertebrae and low back pain. In contrast, and using established diagnostic criteria, we have shown a significantly increased incidence of lumbosacral transitional anomalies in patients with disc prolapse.

The lumbosacral angle is difficult to measure accurately. It varies widely in the normal population and is also affected by factors such as posture and muscle spasm. Given these difficulties and our inability to relate the angle to specific pathology, we are unable to say whether its measurement has any value in clinical practice.

The findings of our study do not argue for increased use of the plain lumbar radiograph as a diagnostic tool and we would agree that it has a limited role in the investigation of low back pain in the individual patient (Waddell 1982). Nevertheless, our findings do support 
the proposition that the occurrence and level of disc prolapse is related to mechanical factors which can be illustrated to some extent on plain radiographs.

The authors are grateful to the orthopaedic consultants in Norwich for allowing their patients to be included in this study, to the Department of Medical Illustration at the Norfolk and Norwich Hospital and to Miss S Yeomans and Mrs S. J. MacLean for their secretarial assistance. They would also like to acknowledge the assistance of $\mathrm{Mr} \mathrm{L}$. Waldron superintendent radiographer at the Norfolk and Norwich Hospital.

No benefits in any form have been received or will be received from a commercial party related directly or indirectly to the subject of this article.

\section{REFERENCES}

Bene E. Measurement of the lumbosacral angle and its clinical significance. $Z$ Rheumatol 1981; 40:149-52.

Fullenlove TM, Williams AJ. Comparative roentgen findings in symptomatic and asymptomatic backs. Radiology 1957; 68:572-4.

MacGibbon B, Farfan HF. A radiologic survey of various configurations of the lumbar spine. Spine $1979 ; 4: 258-66$.
McCulloch JA, Waddell G. Variation of the lumbosacral myotomes with bony segmental anomalies. J Bone Joint Surg [Br] 1980; 62B:475-80.

Mitchell GAG. The significance of lumbosacral transitional vertebrae. Br J Surg 1936; 24:147-58.

Rowe ML. Are routine spine films on workers in industry cost- or riskbenefit effective? J Occup Med 1982; 24:41-3.

Splithof CA. Lumbosacral junction: roentgenographic comparison of patients with and without backache. JAMA 1953;152:1610-3.

Tini PG, Wieser C, Zinn WM. The transitional vertebra of the lumbosacral spine: its radiological classification, incidence, prevalence and clinical significance. Rheumatol Rehabil 1977; 16: $180-5$.

Von Lackum HL. The lumbosacral region: an anatomic study and some clinical observations. JAMA 1924; 82:1 109-14.

Waddell G. An approach to backache. Br J Hosp Med 1982; 28: 187-219.

Wigh RE. The thoracolumbar and lumbosacral transitional junctions. Spine 1980; 5:215-22.

Witt I. A comparative analysis of $x$-ray findings of the lumbar spine in patients with and without lumbar pain. Spine 1984; 9:298-300.

Zinn WM. Die konservative Behandlung des Kreuzschmerzes. Orthopäde 1972; 177-84. 